\title{
Artifical atoms in interacting graphene quantum dots
}

\author{
Wolfgang Häusler ${ }^{1,2}$ and Reinhold Egger ${ }^{1}$ \\ ${ }^{1}$ Institut für Theoretische Physik, Heinrich-Heine-Universität, D-40225 Düsseldorf, Germany \\ ${ }^{2}$ Physikalisches Institut, Albert-Ludwigs-Universität, D-79104 Freiburg, Germany
}

(Dated: October 31, 2018)

\begin{abstract}
We describe the theory of few Coulomb-correlated electrons in a magnetic quantum dot formed in graphene. While the corresponding nonrelativistic (Schrödinger) problem is well understood, a naive generalization to graphene's "relativistic" (Dirac-Weyl) spectrum encounters divergencies and is ill-defined. We employ Sucher's projection formalism to overcome these problems. Exact diagonalization results for the two-electron quantum dot, i.e., the artificial helium atom in graphene, are presented.

PACS numbers: 73.22.-f, 73.21.La, 78.67.Hc
\end{abstract}

The recent spectacular progress in preparing and usefully employing indivual carbon monolayers of graphene [1, 2] continues to stimulate much interest across different scientific communities, including material science, applied physics, chemistry, condensed matter physics and mathematics. Ballistic electronic motion with quantum coherence extending over micrometer distances has been achieved in several experiments, see, e.g., Ref. [3]. The low-energy physics close to a single $K$ point can then be described by a two-component Dirac-Weyl Hamiltonian [2, 4],

$$
H_{0}=v_{\mathrm{F}} \boldsymbol{\sigma} \cdot\left(\boldsymbol{p}-\frac{e}{c} \boldsymbol{A}\right),
$$

suggesting an easily accessible condensed-matter realization of relativistic quantum mechanics. In Eq. (1), $\sigma$ denotes the vector of the first two Pauli matrices for the "isospin" encoding the two sublattices, the Fermi velocity is $v_{\mathrm{F}} \approx 10^{6} \mathrm{~m} / \mathrm{sec}$, and we include a static vector potential $\boldsymbol{A}(\boldsymbol{r})$ describing (possibly inhomogeneous) magnetic fields. Since graphene's effective fine structure constant is $\alpha \approx 1$, present interest has also turned to Coulomb interaction effects [2]. According to recent Monte Carlo simulations [5] and analytical arguments [6], sufficiently strong interactions may even open a sizeable bulk gap in the Dirac fermion spectrum.

Here we study the properties of few Coulombcorrelated electrons confined to a finite-size quantum dot formed in graphene. Using electrostatically formed quantum dots in semiconductor devices, such "artificial atoms" have been intensely studied over the past two decades, both experimentally [7] and theoretically [8]. In graphene dots formed by electrostatic gating, however, carriers can escape due to the (recently observed [9]) Klein tunneling phenomenon, and at best quasi-bound states may appear [10]. An alternative is to employ lithographically defined quantum dots [11], where detailed information on ground- and excited-state properties has been obtained from transport spectroscopy. Unfortunately, the boundary of lithographically fabricated graphene dots is rather disordered and difficult to model [12]. On the other hand, suitable and realizable inhomogeneous magnetic fields can confine Dirac fermions
[13, 14], promising to yield tunable and well-defined magnetic graphene dots.

A more challenging difficulty to theory arises when trying to generalize Eq. (1) to a first-quantized manyparticle description. The first-quantized approach has turned out to be very efficient and convenient for the case of Schrödinger electrons in semiconductor-based artificial atoms [8]. For the "relativistic" graphene case, the problem arises from the unboundedness of Eq. (1), in contrast to the corresponding Schrödinger operator $(\boldsymbol{p}-$ $\left.\frac{e}{c} \boldsymbol{A}\right)^{2} / 2 m^{*}$. While Eq. (11) can still be used within effective single-particle approximations such as the HartreeFock approach [15], variational schemes [16], or densityfunctional theory [17], the full $N$-particle problem (for small $N>1$ ) with Eq. (11) for the kinetic part suffers from the so-called "Brown-Ravenhall disease" [18, 19]. Roughly speaking, the unbounded spectrum allows particles to lose arbitrary amounts of energy by transferring their energy in (real) scattering events to other particles. The resulting divergent density of states prohibits, for example, the direct use of exact diagonalization methods. This difficulty of the Dirac equation has been known for half a century [18]. To "cure" this "disease", we follow a proposal by Sucher [19] and confine the Hilbert space to positive energy eigenstates through suitably defined projectors, cf. Eq. (6) below. While we formulate this approach for the magnetic dot only, the general concepts remain applicable for almost arbitrary graphene dots. The projection method then allows, for instance, to apply numerical techniques to the relativistic $N$-particle problem. In this work, we present exact diagonalization results for the many-body energy spectrum of the artificial helium atom $(N=2)$ in graphene.

Let us first specify the model discussed here (we set $\hbar=$ 1). In cylinder coordinates, we consider the spherical and parabolic magnetic field profile oriented perpendicular to the graphene plane (with $\boldsymbol{A}$ in symmetric gauge),

$$
\boldsymbol{B}(r, \varphi)=\frac{c}{e} \omega_{\mathrm{B}}^{2} r^{2} \boldsymbol{e}_{z}, \quad \boldsymbol{A}(r, \varphi)=\frac{1}{4} \omega_{\mathrm{B}}^{2} r^{3}\left(\begin{array}{c}
-\sin \varphi \\
\cos \varphi \\
0
\end{array}\right)
$$

The inverse length scale $\sqrt{\omega_{\mathrm{B}}}$ tunes the field inhomo- 
geneity. The dimensionless radial coordinate is $\varrho=$ $r \sqrt{\omega_{\mathrm{B}}}$, and energy $(\varepsilon)$ is measured in units of $v_{\mathrm{F}} \sqrt{\omega_{\mathrm{B}}}$. [Physical units are recovered from $\sqrt{B(\varrho) / \text { Tesla }}=$ $\left.\left(v_{\mathrm{F}} \sqrt{\omega_{\mathrm{B}}} / 26 \mathrm{meV}\right) \varrho.\right]$ Such magnetic profiles can be generated with reasonable accuracy using suitable lithographically defined ferromagnetic films deposited on top of the graphene layer after formation of a protective oxide layer [20]. Upper and lower components of eigenspinors $\left|\psi_{\nu}^{(0)}\right\rangle$ to Eq. (11) must then differ by one orbital angular momentum quantum number $(m)$ due to conserved total angular momentum [13]. With real functions $\phi_{m}(\varrho)$ and $\chi_{m+1}(\varrho)$, the radial part of $\left|\psi_{\nu}^{(0)}\right\rangle$ is $\propto\left(\phi_{m}(\varrho) \text {, i } \operatorname{sgn}(\varepsilon) \chi_{m+1}(\varrho)\right)^{T}$, where Eq. (11) yields the radial equations

$$
\left(\begin{array}{cc}
-\varepsilon & \partial_{\varrho}+\frac{m+1}{\varrho}-\frac{\varrho^{3}}{4} \\
-\partial_{\varrho}+\frac{m}{\varrho}-\frac{\varrho^{3}}{4} & -\varepsilon
\end{array}\right)\left(\begin{array}{c}
\phi_{m}(\varrho) \\
\chi_{m+1}(\varrho)
\end{array}\right)=0,
$$

which cannot be solved analytically. We here carry out exact diagonalizations, later on including the Coulomb interaction, and thus solve Eq. (3) numerically. It is convenient to employ the Darwin-Fock states (with integer $n \geq 0$ and the Laguerre polynomials $L_{n}^{|m|}$ ) [8]

$$
\Phi_{n m}^{(\lambda)}(\varrho, \varphi)=\frac{\mathrm{e}^{\mathrm{i} m \varphi}}{\sqrt{2 \pi}} \sqrt{\frac{2 \lambda n !}{(n+|m|) !}} \mathrm{e}^{-\lambda \varrho^{2} / 2}(\sqrt{\lambda} \varrho)^{|m|} L_{n}^{|m|}\left(\lambda \varrho^{2}\right)
$$

as complete orthonormal function set in two dimensions (2D) to expand $\phi_{m}$ and $\chi_{m+1}$ in Eq. (3). In Eq. (4), we have included an additional tunable width parameter $\lambda$, which can be optimized [21] to reduce the number of required basis functions $0 \leq n \leq n_{\max }$ when approximating the $\left|\psi_{\nu}^{(0)}\right\rangle$ to the desired accuracy. Figure 1 displays the resulting eigenenergies as a function of the orbital angular momentum $m$. As expected, the spectrum is electron-hole symmetric, and for $m \geq 0$, a zero-energy level develops. This zero-energy level is non-dispersing (precisely like a quantum Hall level), despite of the inhomogeneous magnetic field which implies the nontrivial $m$-dependence of all other energy levels. Note that for the corresponding Schrödinger case with a parabolic magnetic field, the zero-energy level is absent.

Next we consider $N$ interacting electrons in such a graphene dot. A naive approach is to consider the firstquantized Hamiltonian

$$
H=v_{\mathrm{F}} \sum_{j=1}^{N} \boldsymbol{\sigma}_{j} \cdot\left[\boldsymbol{p}_{j}-\boldsymbol{A}\left(\boldsymbol{r}_{j}\right)\right]+\sum_{i<j} \frac{\alpha v_{\mathrm{F}}}{\left|\boldsymbol{r}_{i}-\boldsymbol{r}_{j}\right|},
$$

where the fine structure constant is $\alpha=e^{2} /\left(\kappa_{0} v_{\mathrm{F}}\right)$. For typical substrate materials, the dielectric constant is $\kappa_{0} \approx 1.4$ to 4.7 , resulting in $\alpha \approx 0.6$ to 2 . We mention in passing that the "Wigner molecule" regime 22] seems out of reach in graphene dots, since both the kinetic and the potential energy show identical scaling when changing the density [23]. Moreover, we neglect the Zeeman term which is very small in graphene [14]. Up to the spin and

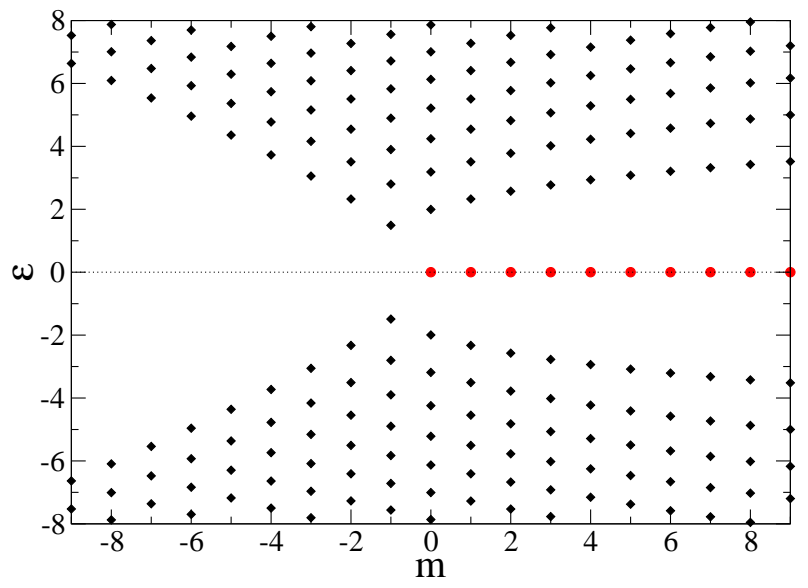

FIG. 1: (Color online) Exact diagonalization results for the single-particle eigenenergies $\varepsilon$ vs orbital angular momentum $m$ in a parabolic magnetic quantum dot in graphene [see Eq. (3)]. The zero-energy levels are indicated as red filled circles, while the other levels are shown as black filled diamonds.

$K$-point indices [24], many-body spinors then have $2^{N}$ components. For the related Schrödinger dot $\left(H_{\mathrm{S}}\right)$, confinement of electrons is usually achieved by a parabolic electrostatic potential [7], and the many-particle description analogous to Eq. (5) simplifies considerably owing to the generalized Kohn theorem 25]. According to this theorem, $H_{\mathrm{S}}=H_{\mathrm{cm}}+H_{\text {rel }}$ separates into two commuting parts describing center-of-mass $\left(H_{\mathrm{cm}}\right)$ and relative $\left(H_{\mathrm{rel}}\right)$ motion. Then $H_{\mathrm{cm}}$ is just a $2 \mathrm{D}$ harmonic oscillator, while $H_{\text {rel }}$ contains all Coulomb interaction effects. In addition, $H_{\text {rel }}$ conserves angular momentum, as does $H_{\mathrm{cm}}$. Taking $N=2$ as example, in effect only a $1 \mathrm{D}$ quantum problem for the radial motion of $H_{\text {rel }}$ remains to be solved. In contrast, Eq. (5) does not benefit from Kohn's theorem, and only the total angular momentum remains conserved as dictated by isotropy. Therefore, while the additional spinor structure already increases the rank of the Hamiltonian matrix in the Dirac-Weyl case by a factor $2^{N}$, the rank grows even more severely because neither $H_{\mathrm{cm}}$ nor a conserved angular momentum of $H_{\text {rel }}$ can be separated off the problem. For $N=2$ (graphene helium), we needed to include states up to $n_{\max } \approx 14$ to reach sufficient accuracy. In addition, contrary to the Schrödinger problem, particles may now exchange relative angular momentum $\Delta m$ through the interaction. Owing to the exponential decay of Coulomb matrix elements with $|\Delta m|$, it is sufficient to take $|\Delta m| \leq 3$, yielding an additional factor $7^{N-1}$ to the matrix size (for $N$ particles). For $N=2$, we then need to include $14^{2} \times 7 \times 2^{2}=5488$ product basis states in total.

Let us then address the more fundamental difficulty arising already for $N=2$ when naively using Eq. (5). A closely related problem has been pointed out by Brown and Ravenhall [18] in a relativistic treatment of the helium atom: the Dirac equation analogous to Eq. (5) does not possess normalizable antisymmetric eigenstates in 
the two-particle Hilbert space. This failure has its origin in the unbounded spectrum of the Dirac Hamiltonian, which allows for unlimited energy exchange among the particles. As a result, the density of two-particle states increases with Hilbert space dimension and ultimately diverges. This causes, e.g., divergent contributions in second-order perturbation theory. In consequence, neither the true Dirac equation nor the two-component variant (1) for graphene allow for naive many-particle generalizations such as Eq. (5). To overcome this deficiency, Sucher [19] proposed to restrict the (anti-symmetrized) product Hilbert space to the positive energy eigenspace for each particle by means of a suitable projector $\Lambda_{+}$. We thus consider a situation with chemical potential $\mu=0^{+}$just above zero, where all non-positive energy (hole) states up to (and including) zero energy are filled. As long as a finite energy gap separates the relevant positive energies (which can then be occupied by the $N$ electrons under consideration) from the filled non-positive energy levels, such a projection seems physically sensible, at least for interaction strengths not exceeding this gap. In natural helium, the mass gap ensures the validity of such an approach. On the other hand, in a homogeneous 2D graphene sheet, weakly interacting fermions are gapless and Sucher's approach does not apply. For our finite-size quantum dot, however, there is a finite gap between $\varepsilon=0$ and the lowest positive-energy level, see Fig. 1. We therefore consider two additional electrons confined in the quantum dot, residing above a filled Dirac sea with $\mu=0^{+}$. The projector proposed by Sucher is expressed as

$$
\Lambda_{+}=\Lambda_{+}^{(1)} \otimes \Lambda_{+}^{(2)}, \quad \Lambda_{+}^{(j)}=\sum_{\nu \in I}\left|\psi_{\nu}^{(j, 0)}\right\rangle\left\langle\psi_{\nu}^{(j, 0)}\right|
$$

where the sum is restricted to strictly positive singleparticle energies $E_{\nu \in I}^{(j, 0)}>0$ (for particle $j=1,2$ ) indexed by $\nu \in I$, see Fig. 1 with corresponding eigenspinors $\left|\psi_{\nu}^{(j, 0)}\right\rangle$. With $H$ in Eq. (5), the projected Hamiltonian $H_{\mathrm{D}}=\Lambda_{+} H \Lambda_{+}$is well-behaved and exhibits a finite density of two-particle states which does not increase with Hilbert space size. Throughout the experimentally relevant regime, $\alpha \lesssim 2$, interactions are not strong enough to induce a breakdown of this projection approach.

We have carried out exact diagonalizations of $H_{\mathrm{D}}$ for $N=2$ using a two-particle product basis of DarwinFock states (4). This basis has the advantage of allowing to analytically express the matrix elements of the two-particle interaction operator in Eq. (5) in terms of finite sums and products, i.e., no quadratures nor truncations of infinite sums are necessary; for the corresponding (lengthy) expressions, see Ref. 26]. Their numerical evaluation involves taking small differences of huge numbers, the latter increasing as $n_{\max }$ ! with the number of kept Darwin-Fock states. We employed algorithms allowing for number manipulations of arbitrary precision and used 30-digits accuracy. The resulting energy spectrum of artificial helium in a magnetic graphene quantum dot is shown in Fig. 2 for conserved total orbital angular

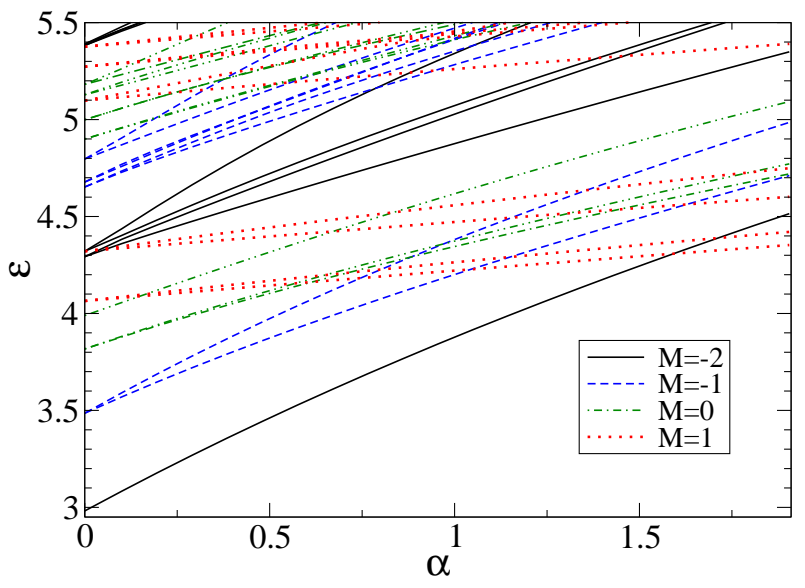

FIG. 2: (Color online) Exact diagonalization results for the energy spectrum of graphene artificial helium vs fine structure constant $\alpha$ for different total angular momenta $M$. States with $M=-2$ are shown as black solid, $M=$ -1 as blue dashed, $M=0$ as green dot-dashed, and $M=1$ as red dotted curves.

momenta $M=m_{1}+m_{2}=-2,-1,0,1$. These values include the ground state for $\alpha<2$. All levels rise when increasing the (repulsive) interaction strength. This also holds true for the hole states (not displayed in Fig. 2), which, however, $\operatorname{cross} \varepsilon=0$ only for $\alpha \gg 2$. For $\alpha<2$, interaction matrix elements indeed remain much smaller than the energy difference $\Delta \varepsilon \simeq 2.98165$ between the lowest two-particle state (for $M=-2$ at $\alpha=0$ ) and the zero-energy level, a posteriori justifying Sucher's approach here. Figure 2 reveals that states with larger total angular momentum $M$, or higher excited states, tend to increase less in energy with $\alpha$ as compared to the $M=-2$ ground state. This is a consequence of the larger spatial extent of excited-state wavefunctions, with a reduced Coulomb repulsion between the electrons. Particularly striking is the shallow increase of the lowest $M=1$ energy level, which even becomes lower in energy than the $M=-2$ level for $\alpha \gtrsim 1.6$. Approximating this level at $\alpha=0$ by Darwin-Fock levels, one of the two particles is seen to have $m=2$ for the lower spinor component, cf. Eq. (3), causing a significantly larger spread of this part of the wavefunction compared to $M=-2,-1,0$. Figure 2 also reveals nontrivial spin physics. In the presence of interactions $(\alpha>0)$, doubly degenerate noninteracting $(\alpha=0)$ energy levels will split into a spin-triplet $(S=1)$ state of lower energy and a spin-singlet $(S=0)$ state of higher energy, in accordance with Hund's rule. The triplet states are approximately (see below) Zeemandegenerate. Singly degenerate $\alpha=0$ levels, such as the $M=-2$ ground state (for small $\alpha$ ), are $S=0$ states and remain unsplit for $\alpha>0$. Thus we expect singlet-triplet ground-state spin transitions to occur within $0<\alpha<2$, as the one seen in Fig. 2 at $\alpha \approx 1.6$.

Finally, we remark on optical transitions between the many-body energy levels in Fig. 2. For the electrostatically defined parabolic Schrödinger quantum dot, 
the generalized Kohn theorem implies that Coulomb interactions can never affect optical transitions because the dipole operator $\sum_{j=1}^{N} \boldsymbol{r}_{j}$ acts exclusively on the eigenspace of $H_{\mathrm{cm}}$. Therefore optical spectra just reflect the harmonic excitations of the center-of-mass motion [8]. However, in our magnetic graphene dot, Kohn's theorem is ineffective and optical transitions between different many-body levels in Fig. 2 are possible, thereby allowing to optically probe interaction physics. Note that magnetic fields are usually assumed homogeneous such that photons cannot change the total spin $S$ of the charged many-particle system in electrical dipole transitions. While this would prohibit all transitions between states with different $S$, the inhomogeneous magnetic field here (slightly) mixes the $S_{z}=0$ components of $S=0$ and $S=1$ levels. We estimate the amount of this mixing by the variation of the Zeeman energy across the spatial extent of the wavefunction compared to the level separations of $H_{\mathrm{D}}$. As a first estimate, compare the Zeeman energy $\Delta_{Z}$ at the maximum of the charge-density distribution with the orbital (Landau) energy $\Delta_{L}$ at this point, $\Delta_{Z} / \Delta_{L}=g \mu_{\mathrm{B}} B / \sqrt{2 e c B} \simeq 10^{-5} \sqrt{B / \text { Tesla. }}$. While this is small, the Zeeman energy variations can easily exceed orbital level separations near spin singlet-triplet degeneracies, e.g., for $\alpha \ll 1$ or close to level crossings in Fig. 2 , resulting in a strong spin mixing. The corresponding transitions are then optically allowed.

To conclude, we have presented the theory of few interacting electrons in a (magnetically confined) graphene quantum dot. The low-energy Dirac-Weyl spectrum of graphene suggests that one can realize relativistic artificial atoms in this setting. While a naive formulation encounters conceptual difficulties related to the unboundedness of the Dirac-Weyl Hamiltonian, by virtue of Sucher's projection operator approach, a consistent and accurate theory can be given. We have presented exact diagonalization results for the energy spectra of artificial helium, where we predict singlet-triplet ground-state spin transitions to occur for $\alpha<2$. Moreover, the reported many-body levels can be experimentally probed by optical spectroscopy. - We thank H. Siedentop for drawing our attention to Refs. [18] and [19], and acknowledge discussions with A. De Martino, K. Ensslin, and T. Heinzel. This work was supported by the DFG (SFB TR/12) and by the ESF network INSTANS.
[1] A.K. Geim and K.S. Novoselov, Nat. Mater. 6, 183 (2007).

[2] A.H. Castro Neto, F. Guinea, N.M.R. Peres, K.S. Novoselov, and A. Geim, Rev. Mod. Phys. 81, 109 (2009).

[3] X. Du, I. Skachko, A. Barker, and E.Y. Andrei, Nat. Nanotech. 3, 491 (2008); K.I. Bolotin, K.J. Sikes, J. Hone, H.L. Stormer, and P. Kim, Phys. Rev. Lett. 101, 096802 (2008).

[4] The true electronic spin is kept implicit, and there is a similar Hamiltonian for the other $K$ point.

[5] J.E. Drut and T.A. Lähde, Phys. Rev. Lett. 102, 026802 (2009); Phys. Rev. B 79, 165425 (2009).

[6] D.V. Khveshchenko, J. Phys. Cond. Matt. 21, 075303 (2009).

[7] L.P. Kouwenhoven, D.G. Austing, and S. Tarucha, Rep. Prog. Phys. 64, 701 (2001).

[8] S.M. Reimann and M. Manninen, Rev. Mod. Phys. 74, 1283 (2002).

[9] N. Standar, B. Huard, and D. Goldhaber-Gordon, Phys. Rev. Lett. 102, 026807 (2009); A.F. Young and P. Kim, Nat. Phys. 5, 222 (2009).

[10] J. Milton Pereira, Jr., V. Mlinar, F.M. Peeters, and P. Vasilopoulos, Phys. Rev. B 74, 045424 (2006); P.G. Silvestrov and K.B. Efetov, Phys. Rev. Lett. 98, 016802 (2007); H.-Y. Chen, V. Apalkov, and T. Chakraborty, ibid. 98, 186803 (2007).

[11] C. Stampfer, J. Güttinger, F. Molitor, D. Graf, T. Ihn, and K. Ensslin, Appl. Phys. Lett. 92, 012102 (2008); L.A. Ponomarenko et al., Science 320, 356 (2008); S. Schnez et al., Appl. Phys. Lett. 94, 012107 (2009); J. Güttinger et al., preprint arXiv:0904.3506.

[12] S. Schnez, K. Ensslin, M. Sigrist, and T. Ihn, Phys. Rev. B 78, 195427 (2008); J. Wurm, A. Rycerz, I. Adagideli, M. Wimmer, K. Richter, and H.U. Baranger, Phys. Rev.
Lett. 102, 056806 (2009).

[13] A. De Martino, L. Dell'Anna, and R. Egger, Phys. Rev. Lett. 98, 066802 (2007); Sol. St. Comm. 144, 547 (2007).

[14] W. Häusler, A. De Martino, T.K. Ghosh, and R. Egger, Phys. Rev. B 78, 165402 (2008).

[15] B. Wunsch, T. Stauber, and F. Guinea, Phys. Rev. B 77, 035316 (2008); M. Ezawa, ibid. 77, 155411 (2008).

[16] I. Romanovsky, C. Yannouleas, and U. Landman, Phys. Rev. B 79, 075311 (2009).

[17] M. Polini, A. Tomadin, R. Asgari, and A.H. MacDonald, Phys. Rev. B 78, 115426 (2008).

[18] G.E. Brown and D.G. Ravenhall, Proc. R. Soc. London Ser. A 208, 552 (1951).

[19] J. Sucher, Phys. Rev. 107, 1448 (1957); Phys. Rev. 109, 1010 (1958); Phys. Rev. A 22, 348 (1980); Int. J. Quantum Chem. 25, 3 (1984).

[20] T. Heinzel, private communication.

[21] In principle, each eigenstate $\left|\psi_{\nu}^{(0)}\right\rangle$ can be individually optimized for $\lambda=\lambda(\nu)$, which can be expressed analytically as a fourth-order root. However, choosing $\lambda=1.7$ for all levels is sufficient for the results presented here.

[22] R. Egger, W. Häusler, C.H. Mak, and H. Grabert, Phys. Rev. Lett. 82, 3320 (1999); 83, 462(E) (1999).

[23] H.P. Dahal, Y.N. Joglekar, K.S. Bedell, and A.V. Balatsky, Phys. Rev. B 74, 233405 (2006).

[24] $K-K^{\prime}$ scattering can be neglected for the long-range Coulomb interaction. Assuming that the interaction does not discriminate among sublattices at long wavelengths, the last term in Eq. (5) is diagonal in isospin space and we can map wave functions between the $K$ points. The spectrum is then independent of how particles are distributed over the two $K$ points.

[25] A.O. Govorov and A.V. Chaplik, JETP Lett. 52, 31 (1990). 
[26] V. Halonen, T. Chakraborty, and P. Pietiläinen, Phys. Rev. B 45, 5980 (1992). 